\title{
Determinación de los niveles de arsénico presentes en sistemas de abastecimiento de agua de las regiones Chorotega y Huetar Norte de Costa Rica, América Central
}

\author{
Arsenic levels present in water supply systems of Chorotega \\ and Huetar Northern Regions of Costa Rica, Central America
}

\author{
Jorge Herrera-Murillo ${ }^{1}$ \\ Diana Mora-Campos ${ }^{2}$ \\ Andrea Suarez-Serrano ${ }^{3}$ \\ María Chaves-Villalobos ${ }^{4}$ \\ Pablo Salas-Jiménez ${ }^{5}$ \\ Alejandra Gamboa-Jiménez ${ }^{6}$ \\ Deivis Anchía-Leitón ${ }^{7}$ \\ Universidad Nacional, Costa Rica
}

1 Coordinador del Laboratorio de Análisis Ambiental, Escuela de Ciencias Ambientales, Universidad Nacional, Costa Rica, jorge.herrera.murillo@una.cr

2 Directora de Calidad del Laboratorio de Análisis Ambiental, Escuela de Ciencias Ambientales, Universidad Nacional, Costa Rica diana.mora.campos@una.cr

3 Directora del Centro de Recursos Hídricos para Centroamérica y el Caribe, Sede Chorotega, Universidad Nacional, Costa Rica, andrea.suarez.serrano@una.cr

4 Investigadora del Laboratorio de Análisis Ambiental, Escuela de Ciencias Ambientales, Universidad Nacional, Costa Rica maria.chaves.villalobos@una.cr

5 Director de Aguas del Laboratorio de Análisis Ambiental, Escuela de Ciencias Ambientales, Universidad Nacional, Costa Rica, pablo.salas.jimenez@una.cr

6 Directora de Análisis Fisicoquímicos del Laboratorio de Análisis Ambiental, Escuela de Ciencias Ambientales, Universidad Nacional, Costa Rica, alejandra.gamboa@una.cr

7 Investigador del Laboratorio de Análisis Ambiental, Escuela de Ciencias Ambientales, Universidad Nacional, Costa Rica, deivis.anchia.leiton@una.cr

Este artículo corresponde a la ponencia presentada en el I Congreso Centroamericano de Ciencias de la Tierra y el Mar, realizado en San José, Costa Rica, del 13 al 16 de noviembre de 2017. 


\title{
Resumen
}

Se determinaron las concentraciones de Arsénico (As) presentes en muestras de agua para uso y consumo humano recolectadas en 106 operadores comunales de sistemas de abastecimiento en las regiones Chorotega y Huetar Norte de Costa Rica durante 2013-2017. A las muestras que registraron concentraciones de As por encima de la norma nacional se le evaluaron los restantes parámetros incluidos en el nivel N2 del Reglamento para agua potable de Costa Rica. La determinación de arsénico se hizo utilizando espectrometría de absorción atómica con horno de grafito. Los principales incumplimientos se registraron en los distritos de Bagaces y Cañas mientras que en la Región Huetar Norte se presentan en Agua Zarcas y Los Chiles. En estos distritos se registra un nivel de incumplimiento de la norma nacional $(10 \mu \mathrm{g} / 1)$ que varía desde $66,7 \%$ en el caso de Los Chiles hasta un $50 \%$ en Cañas. Los sistemas que presentan incumplimientos no registraron variaciones temporales significativas en las concentraciones de As en el periodo 2013-2017. Al analizar los parámetros de correlaciones significativas registradas entre el As y las otras especies analizadas se obtiene información valiosa relacionada con los procesos hidrogeoquímicos que determinan la presencia de este metaloide.

Palabras clave: Arsénico, agua para consumo humano, Costa Rica, Región Chorotega, Región Huetar Norte

\begin{abstract}
Arsenic (As) concentrations in water samples for human consumption and use were determined in 106 communal supply system operators in the Chorotega and Huetar Norte regions of Costa Rica during 2013-2017. The samples that recorded As concentrations above the national standard were evaluated for the remaining parameters included in level N3 of the Regulation for drinking water of Costa Rica. The determination of arsenic was made using atomic absorption spectrometry with graphite furnace. The main non-compliances were registered in the districts of Bagaces and Cañas, while in the Huetar Norte region they are presented in Agua Zarcas and Los Chiles. In these districts there is a level of non-compliance with the national standard $(10 \mu \mathrm{g} / 1)$ that varies from $66.7 \%$ in the case of Los Chiles to $50 \%$ in Cañas. The systems that present breaches by As did not register significant temporal variations in the 2013-2017 period. When analyzing the parameters of significant correlations recorded between the As and the other species analyzed, valuable information is obtained related to the hydrogeochemical processes that determine the presence of this metalloid.
\end{abstract}

Keywords: Arsenic, drinking water, Costa Rica, Chorotega Region, Huetar Norte Region

\section{Introducción:}

El arsénico (As) es un elemento metaloide que se distribuye ampliamente y su presencia en el medio ambiente podría deberse a fuentes naturales provenientes de la erosión de rocas y sedimentos, procesos de formación de minerales con origen hidrotérmico, erupciones volcánicas y actividad geotérmica (Abejón \& Garea, 2015). Adicionalmente, es utilizado a nivel industrial para el tratamiento de la madera, la elaboración de pigmentos y el procesamiento de semiconductores. El arsénico y sus compuestos se acumulan en animales y plantas y se diseminan a través 
de las cadenas alimenticias, dañando el medioambiente ecológico y a los seres humanos (IARC, 2009). Posee varios estados de valencia, variando desde 0 (As), $-3\left(\mathrm{AsH}_{3}\right), \mathrm{a}+3\left(\mathrm{As}_{2} \mathrm{O}_{3}\right) \mathrm{o}+5\left(\mathrm{Na}_{3} \mathrm{AsO}_{4}\right)$ (Liang, 2007). El As en aguas subterráneas naturales, existe como arseniato, As (V) y arsenito, As (III). En condiciones de reducción, la especie predominante es el arsenito $\left(\mathrm{H}_{3} \mathrm{AsO}_{3}\right)$ en un amplio rango de $\mathrm{pH}$. La movilización de arsénico se controla con frecuencia mediante equilibrios de adsorción / desorción y las condiciones de redox y $\mathrm{pH}$ (Smedley, 2008).

La toxicidad de este elemento en los seres humanos depende de sus formas químicas, donde tanto las especies orgánicas como inorgánicas trivalentes son tóxicos más potentes que las pentavalentes (IARC, 2009). Es catalogado como un carcinógeno humano de clase 1 , al cual se encuentran asociados una amplia gama de efectos agudos y crónicos sobre la salud. La principal fuente de exposición al arsénico es el agua potable (Chung et al., 2014), ya que este migra fácilmente del suelo contaminado al agua subterránea dependiendo de las condiciones geoquímicas (Michael, 2013). La exposición a altas concentraciones de este elemento en el agua potable $(>150 \mu \mathrm{g}$ /L) está relacionada con un mayor riesgo de padecer enfermedades crónicas de diversas naturalezas: cardiovasculares, gastrointestinales, vasculares, respiratorios; diabetes mellitus; embarazo adverso y varios tipos de cáncer, incluidos: vejiga, pulmón, piel no melanoma, riñón e hígado (Bardach et al., 2015; Huang et al., 2015; Monrad et al., 2017; Wang et al., 2014).

A nivel latinoamericano, las estimaciones de la Organización Panamericana de la Salud indican que al menos 4500000 de personas están expuestas permanentemente a niveles de arsénico en agua que ponen en riesgo su salud. Así, por ejemplo, en Argentina, Chile, México, Perú, Nicaragua, Bolivia y Brasil, la presencia del arsénico se debe mayoritariamente a factores naturales de origen geológico así como a diversas actividades antropogénicas que incluyen la explotación minera, la refinación y los procesos electrolíticos de producción de metales y en menor proporción la agricultura a través de la utilización de plaguicidas arsenicales orgánicos (Castro de Esparza, 2006)

En el año 2012, el Gobierno de Costa Rica mediante el Decreto Ejecutivo $\mathrm{N}^{\circ} 37072-\mathrm{S}$ declaró emergencia sanitaria para poder atender la problemática generada por la presencia de arsénico en agua para uso y consumo humano en 24 comunidades de la Región Huetar Norte y la 
provincia de Guanacaste, situación que afecta como mínimo a 12 mil personas, dada la toxicidad de este contaminante. Sin embargo, es importante aclarar que la magnitud real del problema se desconoce a ciencia cierta dados los escasos estudios existentes a nivel regional y nacional, así como a la incapacidad económica de los operadores de agua de poder realizar estudios de monitoreo de la calidad del agua que incluyan metales pesados y otros contaminantes orgánicos en forma regular.

En este artículo se presentan los datos de un diagnóstico realizado por el Laboratorio de Análisis Ambiental de la Universidad Nacional en un número representativo de operadores de servicios de abastecimiento de agua ubicados en las regiones Chorotega y Huetar Norte, con el fin de generar información base que permita orientar la toma de decisiones en procura de garantizar la reducción del riesgo de la población que pudiera estar expuesta a este contaminante.

\section{Metodología}

\section{Área de estudio}

La Región Huetar Norte limita al norte con Nicaragua, al sur con la región Central, al este con la región Huetar Atlántica y al oeste con la región Chorotega (Figura 1). Está conformada por los cantones de Sarapiquí de la provincia de Heredia, San Carlos, Guatuso, Los Chiles, Upala y por los distritos de Sarapiquí del cantón de Alajuela, Río Cuarto del cantón de Grecia y Peñas Blancas del cantón de San Ramón, de la Provincia de Alajuela (Zavallos V, 2013). La población de la región es de 304088 habitantes, según el censo del 2011 y posee una extensión de 9803.4 km² $(18 \%$ del territorio nacional), es decir, una densidad poblacional de 31 habitantes por $\mathrm{km}^{2}$, representando el $7.1 \%$ de la población del país. Las mayores concentraciones poblacionales se encuentran en San Carlos, Sarapiquí, y Upala. (Instituto Nacional de Estadística y Censo, 2011).

Posee un clima tropical con una precipitación variable que oscila entre $2000 \mathrm{~mm} /$ año en las llanuras de Guatuso, Upala y Los Chiles y hasta 5 $000 \mathrm{~mm} / \mathrm{año}$, en los macizos volcánicos. Predominan los órdenes de suelos como inceptisol, andisoles, alfisoles y ultisoles. En cuanto a la geomorfología, en la región se encuentran formaciones como Machuca (Oligoceno), Venado (Mioceno Medio) y Aguacate (Neógeno), en las cuales se encuentran lahares de la formación buena vista y piroclastos del volcán Arenal 
en el periodo terciario Inferior y Cuaternario. En la región se encuentran andesitas, basaltos y aglomerados como producto de la actividad volcánica. (Bergoeing, 2007)

Figura 1. Localización del área de estudio para la determinación de As en sistemas de abastecimiento de agua



La Región Chorotega se ubica al norte del país, y sus límites son al norte con Nicaragua, al sur y al oeste con el Océano Pacífico, y al este con la provincia de Puntarenas y región Huetar Norte. En ella se incluye a la Cordillera Volcánica de Guanacaste, las llanuras del Tempisque, los cerros de Nicoya y las llanuras costeras (Figura 1). Tiene una extensión de 10 140 kilómetros cuadrados (20\% del territorio nacional), y está dividido políticamente en once cantones: Liberia, Bagaces, Abangares, Tilarán, Cañas, Santa Cruz, Carrillo, Nicoya, Nandayure, Hojancha y La Cruz, siendo Liberia y Santa Cruz los cantones con mayor cantidad de habitantes. La población total es de 326953 habitantes, es decir, se cuenta con una densidad de población de 32 habitantes por kilómetro cuadrado. 
El clima de la región es tropical seco con dos épocas bien definidas y una precipitación anual promedio que oscila entre los 1500 y $2000 \mathrm{~mm}$. La región se caracteriza por presentar tres unidades de relieve: Cordilleras Volcánicas de Guanacaste y Tilarán, las serranías del complejo de Nicoya y áreas plano onduladas (unidad conformada por depósitos de ignimbritas). Los tipos de rocas que predominan en la región son de origen volcánico y destacan lavas, brechas, tobas e ignimbritas, del Mioceno-Plioceno, las cuales se encuentran cubiertas de cenizas, principalmente, en las partes altas (Kussmaul, 2000).

\section{Muestreo}

Se recolectaron muestras simples de agua para uso y consumo humano en un total de 106 operadores comunales de sistemas de abastecimiento ubicados en las Regiones Chorotega y Huetar Norte durante el período comprendido entre marzo 2013-diciembre 2017 (Figura 2). La distribución de las muestras tomadas se puede observar en la tabla $\mathrm{N}^{\circ} 1$. Cada uno de los sistemas fue encuestado y se procedieron a identificar la naturaleza y cantidad de fuentes de abastecimiento, georreferenciando cada una de ellas.

En cada fuente se colectaron dos muestras, la primera para el análisis de metales en botellas de polietileno de alta densidad, previamente lavadas con detergente, enjuagadas con agua desionizada y por último colocadas en un baño de ácido nítrico al $5 \%$ durante 24 horas. Para su conservación, cada una de estas muestras fue acidificada con $250 \mu \mathrm{L}$ de ácido nítrico (ácido nítrico al $65 \%(\mathrm{v} / \mathrm{v})$ ) por cada $125 \mathrm{~mL}$ de muestra. La segunda muestra se colecto en un recipiente de polietileno de $1 \mathrm{~L}$ para el análisis de otros parámetros fisicoquímicos como: color, turbiedad, conductividad, nitrato, cloruro, sulfato, fluoruro y dureza total. Después de la recolección, ambas muestras fueron rápidamente transportadas en una nevera portátil al laboratorio. En cada uno de los muestreos se incluyeron blancos de transporte y muestreo con el fin de identificar posibles fuentes de contaminación cruzada. 
Figura 2. Distribución geográfica de los operadores de servicios de abastecimiento de agua muestreados.

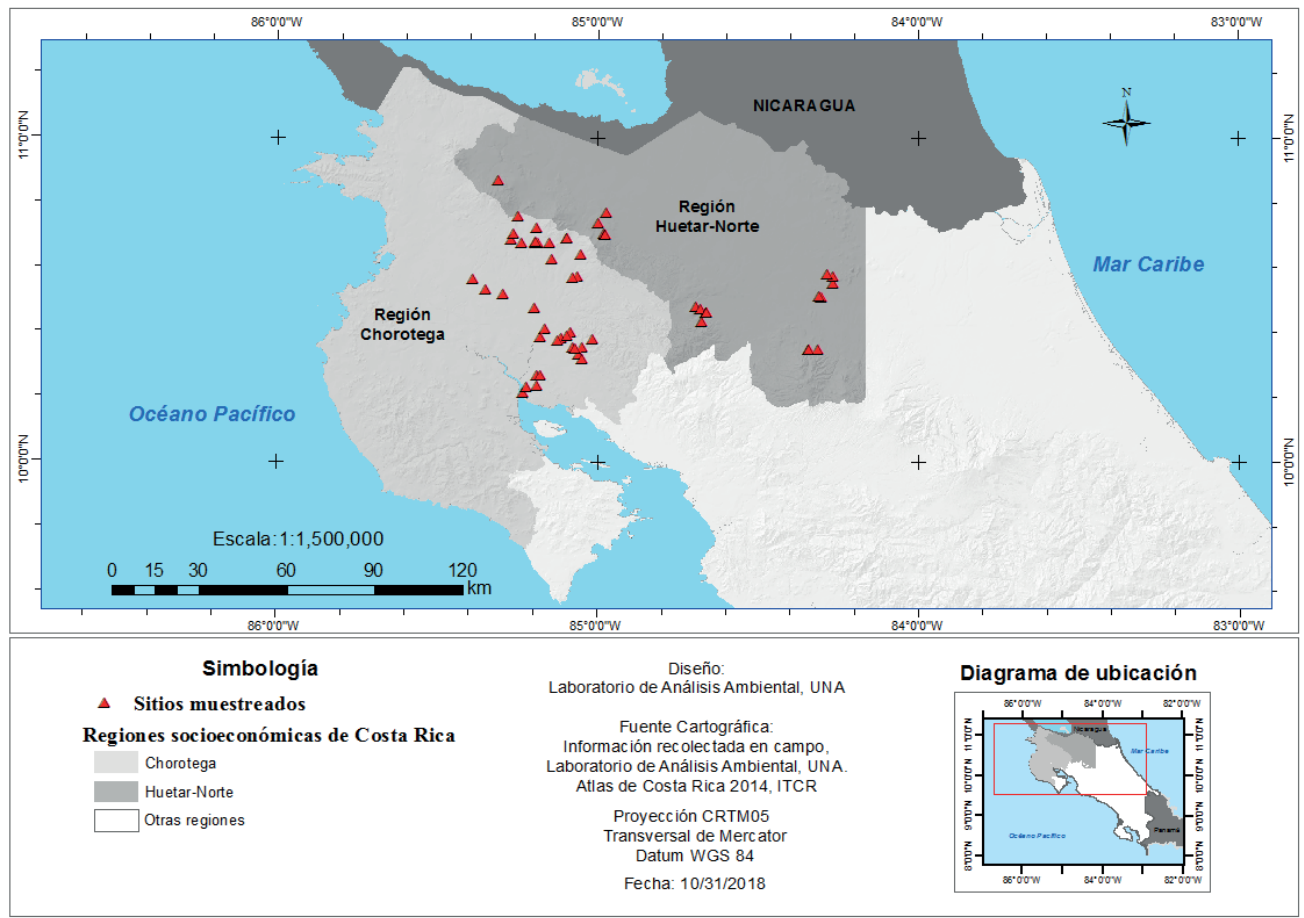

\section{Análisis Químicos:}

La determinación de arsénico y los otros metales (plomo, cobre, cromo, níquel, hierro y aluminio) se hizo utilizando espectrometría de absorción atómica con horno de grafito (Perkin Elmer Analyst 700). Para la cuantificación de las muestras se utilizaron disoluciones estándar de 1000 $\mathrm{mg} / \mathrm{l}$ trazables al NIST, a partir de las cuales se elaboraron curvas de calibración compuestas por 7 patrones en el rango de 5 a $50 \mu \mathrm{g} / \mathrm{l}$. Como mecanismos de control de calidad, se analizaron blancos de reactivos y muestreo, obteniendo como resultado en todos los casos valores menores al límite de detección del método. 
Tabla 1. Distribución de las muestras de agua para uso y consumo humano colectadas de 2013-2017.

\begin{tabular}{|c|c|c|c|c|}
\hline Región & Cantón & Distrito & $\begin{array}{c}\text { Número de entes } \\
\text { operadores }\end{array}$ & $\begin{array}{l}\text { Número de } \\
\text { muestras }\end{array}$ \\
\hline \multirow{11}{*}{ Chorotega } & \multirow{4}{*}{ Bagaces } & Bagaces & 16 & 238 \\
\hline & & La Fortuna & 4 & 40 \\
\hline & & Mogote & 5 & 50 \\
\hline & & Río Naranjo & 2 & 8 \\
\hline & \multirow{5}{*}{ Cañas } & Cañas & 10 & 186 \\
\hline & & Palmira & 2 & 8 \\
\hline & & San Miguel & 3 & 28 \\
\hline & & Bebedero & 2 & 8 \\
\hline & & Porozal & 7 & 28 \\
\hline & Abangares & Abangares & 9 & 36 \\
\hline & Upala & Upala & 1 & 10 \\
\hline \multirow{11}{*}{ Huetar Norte } & \multirow{8}{*}{ San Carlos } & Monterrey & 2 & 8 \\
\hline & & Fortuna & 2 & 8 \\
\hline & & Venecia & 2 & 15 \\
\hline & & Agua Zarcas & 10 & 65 \\
\hline & & Pital & 2 & 18 \\
\hline & & Ciudad Quesada & 7 & 41 \\
\hline & & Florencia & 8 & 45 \\
\hline & & Palmera & 2 & 11 \\
\hline & Guatuso & Guatuso & 3 & 15 \\
\hline & \multirow{2}{*}{ Los Chiles } & Los Chiles & 3 & 29 \\
\hline & & El Amparo & 2 & 17 \\
\hline \multicolumn{3}{|r|}{ Total } & 104 & 912 \\
\hline
\end{tabular}

Fuente: Elaboración propia

La exactitud del análisis químico de metales se comprobó periódicamente mediante el análisis de muestras enriquecidas. Un sesgo general entre -8 y $12 \%$ se obtuvo para las concentraciones de metales medidas en muestras enriquecidas.

El análisis de las especies iónicas $\left(\mathrm{F}^{-}, \mathrm{Cl}^{-}, \mathrm{NO}_{3}^{-}\right.$y $\left.\mathrm{SO}_{4}^{2-}\right)$ se realizó por cromatografía iónica con supresión de conductividad usando un equipo de Dionex ICS- 3000 con una bomba cuaternaria y tubería de microboro. Las muestras se filtraron utilizando una jeringa con un filtro de tamaño de poro de $0,45 \mu \mathrm{m}$. Cada 20 muestras, se preparó una curva de calibración fresca y 
se analizó una disolución de control de calidad de $5 \mathrm{mgl}^{-1}$ preparada a partir de una muestra sintética DIONEX certificada como control de calidad.

Para cada método de análisis empleado se determinaron los límites de detección, preparando 10 muestras de blancos independientes, fortificados a la menor concentración aceptable, los cuales fueron leídos y sus señales fueron interpoladas en una curva de calibración elaborada con patrones ubicados dentro del ámbito lineal del método. El límite de detección se obtuvo como la media de la señal del blanco +3 veces la desviación estándar de la señal de los blancos fortificados. Este enfoque asume que una señal más 3 veces la desviación estándar sobre el valor del blanco podría surgir del blanco menos de $1 \%$ de las veces, y por lo tanto es probable que haya surgido de algo más, como del mensurando. En la tabla 2, se muestra la referencia de los métodos utilizados, así como los respectivos límites de detección.

Tabla 2. Métodos de análisis químicos utilizados con sus respectivos parámetros de mérito.

\begin{tabular}{|c|c|c|c|}
\hline Analito & Método & $\begin{array}{l}\text { Límite de } \\
\text { detección }\end{array}$ & $\begin{array}{c}\text { Límite de } \\
\text { Cuantificación }\end{array}$ \\
\hline Arsénico & \multirow{5}{*}{$\begin{array}{l}\text { SMEWW-APHA-AWWA-WEF: } 3113 \text { B; } \\
\text { Absorción atómica por horno (GFAAS) }\end{array}$} & $1,0 \mu \mathrm{g} / 1$ & $3,0 \mu \mathrm{g} / 1$ \\
\hline Plomo & & $2,0 \mu \mathrm{g} / 1$ & $4,0 \mu \mathrm{g} / 1$ \\
\hline Cobre & & $0,7 \mu \mathrm{g} / \mathrm{l}$ & $0,9 \mu \mathrm{g} / 1$ \\
\hline Aluminio & & $0,9 \mu \mathrm{g} / 1$ & $2,0 \mu \mathrm{g} / 1$ \\
\hline Hierro & & $1,0 \mu \mathrm{g} / 1$ & $2,0 \mu \mathrm{g} / 1$ \\
\hline Sodio & \multirow{5}{*}{$\begin{array}{l}\text { SMEWW-APHA-AWWA-WEF: } 3111 \text { B; } \\
\text { Absorción atómica por llama (FAAS) }\end{array}$} & $0,02 \mathrm{mg} / \mathrm{l}$ & $0,07 \mathrm{mg} / \mathrm{l}$ \\
\hline Potasio & & $0,1 \mathrm{mg} / \mathrm{l}$ & $0,2 \mathrm{mg} / 1$ \\
\hline \begin{tabular}{|l|} 
Calcio \\
\end{tabular} & & $0,01 \mathrm{mg} / \mathrm{l}$ & $0,1 \mathrm{mg} / 1$ \\
\hline Magnesio & & $0,09 \mathrm{mg} / \mathrm{l}$ & $0,10 \mathrm{mg} / \mathrm{l}$ \\
\hline Zinc & & $0,06 \mathrm{mg} / \mathrm{l}$ & $0,10 \mathrm{mg} / \mathrm{l}$ \\
\hline Fluoruro & \multirow{4}{*}{$\begin{array}{l}\text { SMEWW-APHA-AWWA-WEF: } 4110 \text { B; } \\
\text { Cromatografía de Iones }\end{array}$} & $0,08 \mathrm{mg} / \mathrm{l}$ & $0,09 \mathrm{mg} / \mathrm{l}$ \\
\hline Cloruro & & $0,09 \mathrm{mg} / \mathrm{l}$ & $0,20 \mathrm{mg} / \mathrm{l}$ \\
\hline Nitrato & & $0,09 \mathrm{mg} / \mathrm{l}$ & $0,15 \mathrm{mg} / \mathrm{l}$ \\
\hline Sulfato & & $0,1 \mathrm{mg} / \mathrm{l}$ & $0,3 \mathrm{mg} / \mathrm{l}$ \\
\hline Conductividad & $\begin{array}{l}\text { SMEWW-APHA-AWWA-WEF: } 2510 \text { B; } \\
\text { Electroquímico }\end{array}$ & NA & NA \\
\hline Turbiedad & $\begin{array}{l}\text { SMEWW-APHA-AWWA-WEF: } 2130 \text { B; } \\
\text { Nefelométrico }\end{array}$ & NA & NA \\
\hline Color & $\begin{array}{l}\text { SMEWW-APHA-AWWA-WEF: } 2120 \mathrm{C} \\
\text { Espectrofotometría UV-Vis }\end{array}$ & $0,02 \mathrm{UC}$ & $0,08 \mathrm{UC}$ \\
\hline
\end{tabular}

Fuente: Elaboración propia 


\section{Resultados}

En la tabla 3 se presenta la distribución de los resultados obtenidos de arsénico para los sistemas evaluados. Tal como se puede apreciar en la Región Chorotega, los principales incumplimientos se registraron en los distritos de Bagaces y Cañas mientras que en la Región Huetar Norte se presentan en Agua Zarcas y Los Chiles. En estos distritos se registra un nivel de incumplimiento de la norma nacional $(10 \mu \mathrm{g} / \mathrm{l})$ que varía desde 66,7 $\%$ en el caso de Los Chiles hasta un $50 \%$ en Cañas. Al analizar la geología de ambas regiones, se puede visualizar que los sitios que presentan incumplimientos en Bagaces y Cañas se ubican sobre depósitos de ignimbritas del cuaternario (Figura 3), mientras que los localizados en Los Chiles corresponden a formas de denudación, siendo así que las muestras con concentraciones mayores a $10 \mu \mathrm{g} / \mathrm{l}$, se localizan sobre estructuras geológicas de origen volcánico. Al correlacionar la geología con los sistemas de fallas en la zona de estudio, se identifica que las muestras de Los Chiles se ubican sobre un sistema de fallas asociados al Escarpe de Hess. Mientras que en el caso de Bagaces y Cañas, de igual forma, los puntos con valores más altos en las concentraciones de arsénico se ubican cerca de fallamientos geológicos locales.

Como parte del desarrollo del presente estudio, en aquellos sistemas donde se registraban incumplimientos en los niveles de As, se monitorearon varias veces a lo largo de la vigencia del proyecto con el fin de analizar las posibles variaciones temporales. Tal como se puede apreciar en la tabla 4, las concentraciones de As registradas en algunos de los sistemas que presentan incumplimientos tienden a ser relativamente estables en el tiempo. 
Jorge Herrera-Murillo - Diana Mora-Campos - Andrea Suarez-Serrano - María Chaves-Villalobos Pablo Salas-Jiménez - Alejandra Gamboa-Jiménez - Deivis Anchía-Leitón

Determinación de los niveles de arsénico presentes en sistemas de abastecimiento de agua de las regiones Chorotega y Huetar Norte de Costa Rica, América Central

Figura 3. Distribución de los sitios de muestreo que presentan incumplimientos en la concentración de As de acuerdo con su geomorfología.

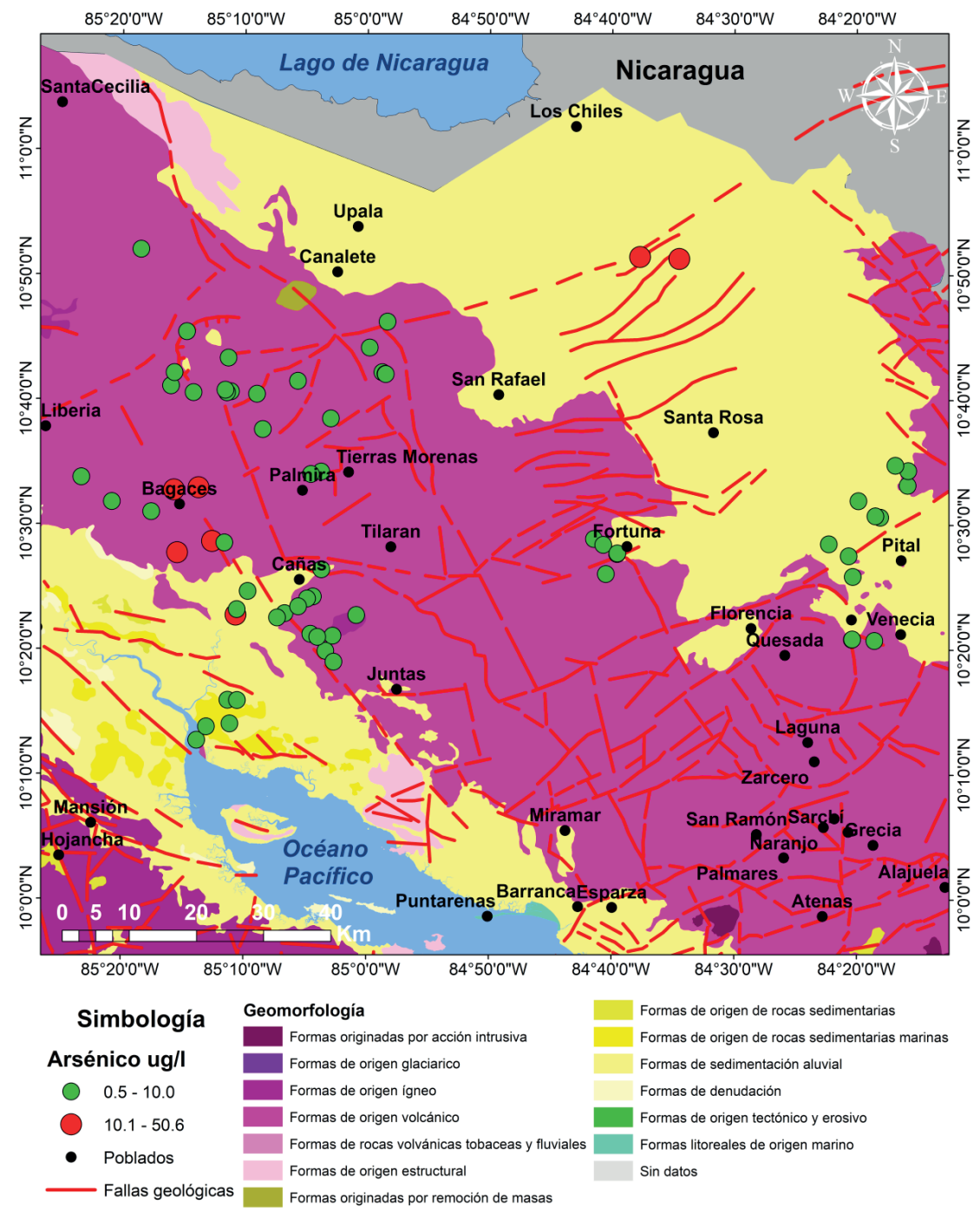

Proyección WGS84, Datum: DWGS84, Fuente: Laboratorio Analisis Ambiental de la Universidad Nacional, Atlas Costa Rica 2014. Elaboración: Geog. Carlos Méndez. 2018. 





Lo anterior pudiera ser un indicativo del origen natural del As encontrado, ya que la geoquímica del agua, así como la composición mineral de los materiales que rodean el acuífero juegan un rol fundamental en los procesos que determinan la concentración del As en las aguas subterráneas. En general, la química de los sistemas de agua subterránea es lenta en cambiar, cuando dominan exclusivamente procesos naturales en comparación con el comportamiento de las fuentes antropogénicas de contaminación.

Tabla 4. Distribución temporal de los niveles de Arsénico presente en fuentes de abastecimiento de una muestra de operadores de servicio de agua para uso y consumo humano en las Regiones Chorotega y Huetar Norte, 2013-2017.

\begin{tabular}{|c|c|c|c|c|c|c|c|c|c|}
\hline \multirow{2}{*}{ Región } & \multirow{2}{*}{ Cantón } & \multirow{2}{*}{ Distrito } & \multirow{2}{*}{$\begin{array}{c}\text { Nombre del } \\
\text { operador }\end{array}$} & \multirow{2}{*}{$\begin{array}{c}\text { Tipo de } \\
\text { fuente de } \\
\text { abastecimiento }\end{array}$} & \multicolumn{5}{|c|}{ Concentración de As $(\mu \mathrm{g} / \mathrm{l})$} \\
\hline & & & & & 2013 & 2014 & 2015 & 2016 & 2017 \\
\hline \multirow[t]{7}{*}{ Chorotega } & \multirow[t]{5}{*}{ Bagaces } & \multirow[t]{5}{*}{ Bagaces } & Falconiana & Pozo & 24 & 26 & 28 & 25 & - \\
\hline & & & Aguacaliente & Pozo & 14 & 16 & 17 & 16 & - \\
\hline & & & El Arbolito & Pozo & 20 & 15 & 18 & 15 & - \\
\hline & & & El Chile & Pozo & 17 & 14 & 12 & 14 & - \\
\hline & & & El Recreo & Pozo & 36 & 40 & 41 & 44 & - \\
\hline & \multirow[t]{2}{*}{ Cañas } & \multirow[t]{2}{*}{ Cañas } & Bebedero & Pozo & - & 15 & 16 & 18 & 21 \\
\hline & & & Javillas & Pozo & 14 & 15 & 17 & 16 & \\
\hline \multirow[t]{5}{*}{$\begin{array}{l}\text { Huetar } \\
\text { Norte }\end{array}$} & \multirow[t]{2}{*}{$\begin{array}{l}\text { San } \\
\text { Carlos }\end{array}$} & \multirow[t]{2}{*}{$\begin{array}{l}\text { Agua } \\
\text { Zarcas }\end{array}$} & $\begin{array}{l}\text { Vuelta de } \\
\text { Kooper }\end{array}$ & Pozo & - & 39 & 31 & 34 & 36 \\
\hline & & & Los Chiles & Pozo & - & 44 & 42 & 47 & - \\
\hline & \multirow{3}{*}{$\begin{array}{l}\text { Los } \\
\text { Chiles }\end{array}$} & \multirow{3}{*}{$\begin{array}{l}\text { Los } \\
\text { Chiles }\end{array}$} & Santa Cecilia & Pozo & - & 16 & 18 & 16 & - \\
\hline & & & Cristo Rey & Pozo & - & 13 & 16 & 17 & - \\
\hline & & & Los Chiles & Pozo & - & 18 & 15 & 15 & - \\
\hline
\end{tabular}

Fuente: Elaboración propia

Para cada uno de los sistemas monitoreados que registraron incumplimientos, se realizó una evaluación del riesgo producto de las concentraciones de As encontradas para niños de 12 años de edad, utilizando el factor para la exposición no carcinogénica, de acuerdo con la siguiente ecuación (Baig et al., 2011):

$$
H Q=\frac{E D I}{R f D}
$$


Donde RfD es el valor de referencia de toxicidad oral para As $(3,04$ x $10^{-4} \mathrm{mg} / \mathrm{kg}$ / día), y la dosis estimada de ingesta diaria (EDI) ( $\mathrm{mg} / \mathrm{kg} /$ día) se calculó de la siguiente manera:

$$
E D I=\frac{(C \times I R \times E F \times E D)}{(B W \times A T)}
$$

Donde C es la concentración de As en agua (mg / L), IR la tasa de admisión (L/día), EF la frecuencia de exposición (día /año), ED la duración de la exposición (año), BW el peso corporal (kg), y AT en el tiempo promedio de edad (días). De acuerdo con la literatura, no se observan efectos adversos para la salud si la HQ calculada es < 1 (USEPA, 1998). El dato de peso corporal de los niños de 12 años se obtuvo del Primer Censo Escolar Peso y Talla de Costa Rica: año 2016, realizado por el Ministerio de Educación Pública. En la Tabla 5 se muestra que para todos los operadores de servicios de abastecimiento donde se registraron excedencias a los límites contenidos en la normativa nacional, se reportan valores de HQ que podrían acarrear efectos adversos para la salud de los niños por ingesta del agua.

Para aquellos sistemas de abastecimiento, cuyas muestras de agua presentaron incumplimientos por As, en las regiones de estudio se realizó el análisis de los restantes parámetros fisicoquímicos incluidos en el nivel N2 del Reglamento para la calidad del Agua Potable de Costa Rica, Decreto 38924-S indicados en la tabla 2. Al aplicar la prueba de AndersonDarling, para evaluar la normalidad de todos los parámetros medidos se encontró que el 100\% siguen una distribución no normal $(\mathrm{p}<0,05)$. En la Tabla 6, se muestran los principales resultados de los restantes parámetros químicos analizados en las muestras con incumplimientos.

La principal fuente de iones disueltos en la mayoría de los sistemas acuáticos es la meteorización de los minerales formadores de rocas, sin embargo, las fuentes atmosféricas y antropogénicas contribuyen a la forma en la que estos constituyentes se encuentran presentes en los recursos hídricos (Zhang et al., 2008). De acuerdo con los resultados del análisis, se puede observar que el orden de la mayor abundancia de aniones es $\mathrm{Cl}^{-}>$ $\mathrm{SO}_{4}^{2->} \mathrm{NO}_{3}^{-}$en las muestras de aguas recolectadas en la región Chorotega 





mientras que en Huetar Norte la secuencia corresponde a $\mathrm{SO}_{4}{ }^{2-}>\mathrm{Cl}^{-}>\mathrm{NO}_{3}{ }^{-}$. La secuencia de cationes es $\mathrm{Na}^{+}>\mathrm{K}^{+}>\mathrm{Mg}^{2+}>\mathrm{Ca}^{2+}$ en las muestras de agua para ambas regiones. El calcio y el magnesio son elementos abundantes en las aguas subterráneas y son, además, los principales contribuyentes a la dureza del agua.

La principal fuente de estos elementos en las aguas subterráneas es la erosión de las rocas, como la piedra caliza y la dolomita, y minerales como la calcita, el yeso y la magnesita (Kumar et al., 2015). Al determinar los patrones de correlación de Spearman entre las variables medidas, se

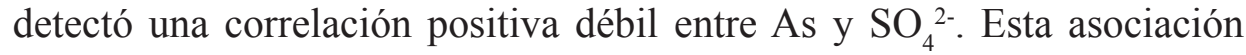
sugiere que el As disuelto presente en las muestras de agua no se pudo haber originado de la oxidación de minerales de sulfuro en los acuíferos (Choudhury et al., 2017). Otras correlaciones muy débiles se registraron entre As-Fe y As-Mn, que son componentes sensibles a redox, lo que puede usarse para inferir que la movilización de As tiene lugar a través de diferentes mecanismos, tales como la reducción del $\mathrm{SO}_{4}{ }^{2-}$ y la desorción de óxidos de metal (óxidos de $\mathrm{Fe}$ y $\mathrm{Mn}$ ) a las condiciones de $\mathrm{pH}$ presente en las muestras. La correlación entre As y $\mathrm{F}^{-}$resulto ser significativa $(0,748)$ lo que puede ser explicado debido al hecho de algunos iones coexistentes en el agua pueden inhibir o facilitar la adsorción de arsénico. De esta forma los aniones como $\mathrm{Cl}^{-}, \mathrm{F}^{-}, \mathrm{SO}_{4}{ }^{2-}$ y $\mathrm{HCO}_{3}^{-}$pueden aumentar el contenido de arsénico en las aguas subterráneas (Baig et al., 2011). . 







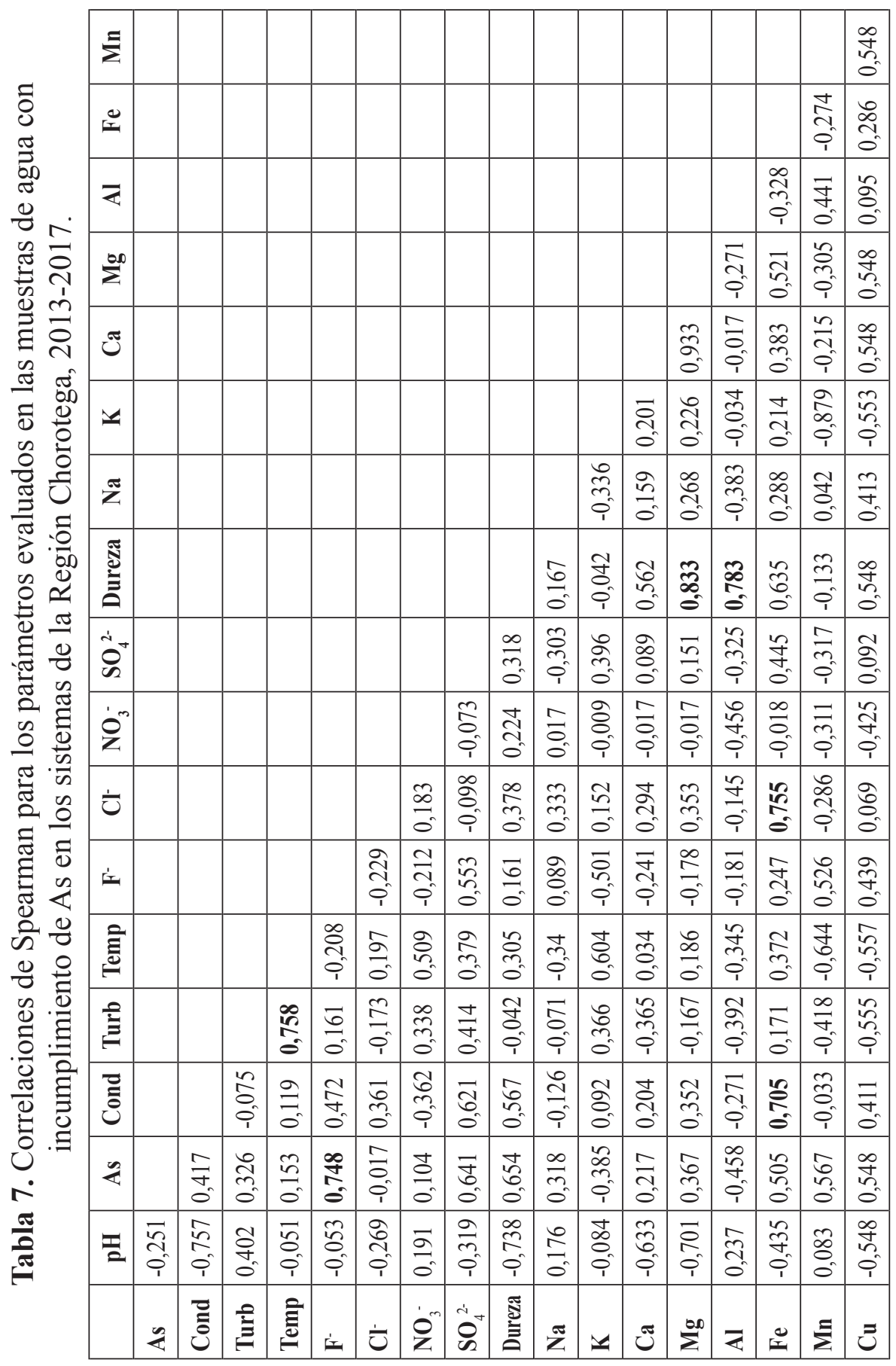

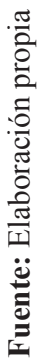




\section{Conclusiones}

Las concentraciones de As encontradas en algunos entes operadores de sistemas de abastecimiento de aguas de las regiones Chorotega y Huetar Norte superan la norma nacional de $10 \mu \mathrm{g} / \mathrm{l}$ y representan un riesgo significativo a la salud para aquellos habitantes que la consumen, especialmente para los niños. Sin embargo, por informes del AyA desde el año 2010 se inició la determinación de As en algunos de los sitios de estudio, esto quiere decir que el riego por exposición (e ingesta) de agua para consumo contaminada podría ser mucho mayor que la estimada. Por lo que, es recomendable realizar estudios epidemiológicos en las zonas para conocer realmente el estado de salud actual de las personas expuestas, sobre todo aquellas más vulnerables.

El patrón de correlaciones registrado con otros parámetros medidos podría indicar que la movilización de As tiene lugar a través de diferentes mecanismos, tales como la reducción del $\mathrm{SO}_{4}^{2-}$ y la desorción de óxidos de metal (óxidos de Fe y $\mathrm{Mn}$ ) a las condiciones de $\mathrm{pH}$ presente en las muestras. Adicionalmente, el comportamiento de las concentraciones de As en las fuentes a través del tiempo hace presumir que estas son determinadas por procesos naturales en ausencia de fuentes antropogénicas importantes.

Se considera que para el desarrollo de futuras investigaciones sería conveniente indagar en las características geoquímicas y la variabilidad espacial de materiales geológicos y edáficos, así como generar conocimientos sobre los mecanismos y las formas de especiación del ion arsénico en el agua subterránea.

\section{Referencias}

Abejón, R. \& Garea, A. (2015). A bibliometric analysis of research on arsenic in drinking water during the 1992-2012 period: An outlook to treatment alternatives for arsenic removal. Journal of Water Process Engineering. 6, 105-119.

Baig, J.A., Kazi, T.G., Shah, A.Q., Afridi, H., Khan, S., Kolachi, N., Kandhro, G. \& Wadhwa, S. (2011). Evaluation of toxic risk assessment of arsenic in male subjects through drinking water in southern Sindh Pakistan. Biological Trace of Elements Research, 143, 772-786.

Bardach, A.E., Ciapponi, A., Soto, N.; Chaparro, M.R., Calderón, M. $\&$ Briatore, A. (2015). Epidemiology of chronic disease related to 
arsenic in Argentina: a systematic review. Science of the Total Environment, 538, 802-816.

Castro, M. (2006) Presencia de arsénico en el agua de bebida en América Latina y su efecto en la salud pública. Centro Panamericano de Ingeniería Sanitaria y Ciencias del Ambiente (CEPIS-SB/SDE/OPS). Congreso International Ciudad de México, 20-24 junio 2006.

Choudhury, R., Mahanta, C., Verma, S. \& Mukherjee. A. (2017). Arsenic distribution along different hydrogeomorphic zones in parts of the Brahmaputra River Valley, Assam (India). Journal of Hydrogeology 25,1153-1163.

Chung, J.Y., Seung, Y. \& Young, H. (2014). Environmental source of arsenic exposure. Journal of Prevention Medical and Public Health, 47, 253-257

Huang, L., Wu, H. \& van der Kuijp, T.J. (2015). The health effects of exposure to arsenic-contaminated drinking water: a review by global geographical distribution. International Journal of Environmental Health Research, 25, 432-452

International Agency for Research on Cancer (IARC). (2009) Arsenic and Arsenic Compounds. IARC Monographs on the Evaluation of Carcinogenic Risks to Humans Vol. 100C-6. International Agency of Research on Cancer, Lyon, France.

Kumar, S.K., Logeshkumaran, A., Magesh, N.S., Godson, P.S. \& Chandrasekar, N. (2015) Hydrogeochemistry and application of water quality index (WQI) for groundwater quality assessment, Anna Nagar, part of Chennai City, Tamil Nadu, India. Applied Water Sciences 5:335-343

Liang, Y. (2007) Transformation and Bioavailability of Arsenic in Soil. Huazhong Agricultural University.

Michael, H.A. (2013). An arsenic forecast for China. Science, 341, 852-853

Monrad, M., Ersbøll, A.K., Sørensen, M., Baastrup, R., Hansen, B., Gammelmark, A. (2017) Low-level arsenic in drinking water and risk of incident myocardial infarction: a cohort study. Environmental Research, 154, 318-324.

Smedley, P.L. (2008). Sources and distribution of arsenic in groundwater and aquifers. C.A.J. Appelo (Ed.), Arsenic in Groundwater: A World 
Problem, Proceedings of the IAH. Seminar, Utrecht, Netherlands (2008), 4-32

Wang, W., Cheng, S. \& Zhang, S. (2014). Association of inorganic arsenic exposure with liver cancer mortality: a meta-analysis. Environmental Research, 135, 120-125.

United States Environmental Protection Agency (US EPA) (1998). Integrated Risk Information System: Arsenic, Inorganic. CASRN; 7440-38-2.

Zhang, Q., Kang, S., Wang, F., Li, C. \& Xu, Y. (2008) Major ion geochemistry of Nam Co Lake and its sources, Tibetan Plateau. Aquatic Geochemistry 14(4):321-336 